\title{
Improving immunization strategies in patients with inflammatory bowel disease
}

\author{
Maham Farshidpoura, Aline Charabaty ${ }^{b}$, Mark C. Mattar ${ }^{b}$ \\ Banner University Medical Center, University of Arizona, Tucson; MedStar Georgetown University Hospital, \\ Washington, DC, USA
}

\begin{abstract}
Patients with inflammatory bowel disease (IBD) are susceptible to varieties of opportunistic infections due to immunological changes in the setting of their disease and drug-induced immunosuppression. Even though numerous infections can be prevented by vaccine, vaccination in IBD patients is inadequate. Data showed only $9 \%$ were vaccinated against pneumococcal infection and $28 \%$ described commonly receiving influenza vaccine. This review article discusses the recent immunizations against influenza virus; pneumococcal infection; human papilloma virus; tetanus, diphtheria and pertussis; measles, mumps and rubella; varicella zoster; and herpes zoster for individuals diagnosed with IBD and those patients with drug-related immunosuppression. In addition, this review discusses concerns about IBD patients planning to travel abroad. Immunization status and screening for opportunistic infection need to be addressed in IBD patients at the time of diagnosis and they should be vaccinated accordingly. Generally, standard vaccination strategies should be pursued in IBD patients, although live vaccines should be avoided while they are not immunocompetent.
\end{abstract}

Keywords Inflammatory bowel disease, opportunistic infections, immunization

Ann Gastroenterol 2019; 32 (2): 1-10

\section{Introduction}

Inflammatory bowel diseases (IBD) are a group of chronic inflammatory conditions of the colon and small intestinal tract [1]. Immunomodulators and biologic agents are approved for treating this group of patients and current data support their introduction early in the disease course [2]. Biologic medications and immunomodulators, or a combination of both, are used as the maintenance therapy in IBD [3]. Immunosuppression increases the risk of infections, some of which are preventable with routine immunization [4]. IBD patients on immunosuppressive treatment have a considerably

${ }^{a}$ Department of Inpatient Medicine, Banner University Medical Center, University of Arizona, Tucson (Maham Farshidpour); ${ }^{b}$ Division of Gastroenterology, MedStar Georgetown University Hospital, Washington, DC, USA (Aline Charabaty, Mark C. Mattar), USA

Conflict of Interest: None

Correspondence to: Maham Farshidpour, MD, Clinical Assistant Professor, Department of Medicine-University of Arizona, Banner University Medical Center-Tucson, 1501 N. Campbell Ave-Room 6324, Tucson, AZ 85724, USA,

e-mail: Maham.Farshidpour@bannerhealth.com

Received 28 September 2018; accepted 11 November 2018; published online 15 January 2019

DOI: https://doi.org/10.20524/aog.2019.0351 weaker reaction to routine vaccinations. The greatest effect is seen in patients on a combination of anti-tumor necrosis factor (TNF) and immunosuppressive therapy [5]. Given the risk of vaccine-related infection, live vaccines are contraindicated in immunodeficient IBD patients (Table 1) [6].

Data have shown poor counselling about vaccinations by gastroenterologists or primary care physicians [7]. Prior studies have proven that physician counseling is a strong predictor of being vaccinated and other preventive care interventions [8]. Therefore, these findings draw attention to the need for a thorough and organized assessment of immunization status at the time of diagnosis of IBD or prior to starting any biologic agents. The aim of this review is to improve gastroenterologists' knowledge of the importance of preventive healthcare within the IBD patient population.

\section{Materials and Methods}

MEDLINE records were explored through PubMed with search strategies using search keywords "IBD”, "immunization", "vaccination recommendation", "influenza", "Europe”, "HPV”, "pneumococcal”, "herpes zoster", "varicella", “Tdap" and "MMR" to identify studies published between the years 1987 and 2018. Articles were selected from case-control studies, randomized trails, cohort research, and case reports. In addition, abstracts of conferences from important congresses in 
the gastroenterology field, United European Gastroenterology Week, and the European Crohn's and Colitis Organisation were searched. Adults with IBD receiving any vaccine type and at any dose were included. Studies related to non-humans or not in the English language were excluded from our review. Abstracts of the articles found by the preliminary search were reviewed by the authors for pertinence to IBD, and all potentially related data were selected and evaluated in detail.

\section{Definition of immunocompromised in IBD}

The criteria for impaired immune systems in IBD patients are: 1) patients on glucocorticoid therapy $\geq 20 \mathrm{mg}$ of prednisone for longer than 2 weeks; 2) patients taking immunomodulators, including azathioprine, mercaptopurine and/or methotrexate, calcineurin inhibitors or anti-TNF (infliximab, adalimumab or others); 3) undernourished patients and patients with any condition leading to impaired immune systems, such as asplenia or human immunodeficiency virus infection [4,9]. Another classification specifies high or low levels of immunosuppression according to the strength of the immunosuppressive agents (Table 2) [10].

Table 1 Contraindicated vaccines in patients with inflammatory bowel disease [10,102-107]

\begin{tabular}{l}
\hline Live, attenuated influenza (intranasal vaccine) \\
Varicella zoster vaccine, Herpes zoster (live zoster vaccine) \\
Yellow fever vaccine \\
Measles-mumps-rubella vaccine \\
Smallpox vaccine \\
Tuberculosis bacillus Calmette-Guérin vaccine \\
Polio live oral vaccine \\
Anthrax vaccine \\
\hline
\end{tabular}

Table 2 Level of immunosuppression based upon strength of immunosuppressive medication [10]

High-level immunosuppression

Treatment with glucocorticoids (prednisone $>20 \mathrm{mg} /$ day for $\geq 2$ weeks and within 3 months of stopping therapy)

Treatment with 6-mercaptopurine, azathioprine, or methotrexate compared with those with low-level immunosuppression (described below) or discontinuation within 3 months

Treatment with adalimumab, certolizumab pegol, golimumab, infliximab, natalizumab, or vedolizumab, or recent discontinuation within 3 month

Low-level immunosuppression

Treatment with lower total daily doses of corticosteroids compared with those with high-level immunosuppression for more than 14 days

Patients receiving methotrexate $(<0.4 \mathrm{mg} / \mathrm{kg} /$ week $)$, azathioprine $(<3.0 \mathrm{mg} / \mathrm{kg} /$ day $)$, or mercaptopurine $(<1.5 \mathrm{mg} / \mathrm{kg} /$ day $)$

\section{Screening test for infectious disease}

The use of biological medications and immunomodulators for IBD is connected to an increased risk of opportunistic infections. Therefore, screeningforimmunity to seriousinfection is recommended, but compliance with these recommendations is unknown. It is essential that gastroenterologists involved in IBD care execute a vigilant investigation for infectious disease before starting immunomodulation. Vigilant screening allows the physician to avoid having to stop a biological medication because of the presence of infections with the risk of recurrence of the underlying disease [11] (Table 3).

\section{Rate of infection in IBD patients}

The risk of opportunist infections is very high in IBD patients. Kirchgesner et al in 2018 showed that, among 190,694 patients with IBD, serious infections occurred in 8561 of them, while 674 patients were dealing with opportunistic infections. The investigators reported that combination therapy was accompanied by higher risks of serious infection (hazard ratio [HR] 1.23, 95\% confidence interval [CI] 1.05-1.45) and opportunistic infection (HR 1.96, 95\%CI 1.32-2.91), compared with anti-TNF monotherapy [12]. Reactivation of the hepatitis $\mathrm{B}$ virus (HBV) has been documented at rates of $16-36 \%$ in IBD patients with HBsAg-positive. Longstanding use (defined as more than 3 months) of immunosuppressive therapy and combination therapy without being immunized with antiviral vaccine prophylactically are associated with the risk of HBV reactivation [13]. Huang et al showed that the rate of hepatitis $\mathrm{C}$ virus (HCV) infection in patients with IBD was not statistically different from that in the general population. Among 714 patients with IBD, the rate of $\mathrm{HCV}$ infection was $0.42 \%$ compared with $0.36 \%(\mathrm{P}=0.80)$ in non-IBD individuals. This outcome was in line with another study conducted in Italy $[14,15]$. The latest data indicate that IBD patients have a $1.65 \%$ chance of developing a tuberculosis infection, even after latent tuberculosis infection screening, before the initiation of anti TNF- $\alpha$ therapy [16].

\section{Vaccination rate in IBD patients}

The vaccination rate among IBD patients is still suboptimal. A survey by Melmed et al showed that, among 146 IBD patients, only $41(28 \%)$ had received an influenza vaccine and 13 (9\%) reported being vaccinated against pneumococcal infection with a history of application of immunosuppressive agents. A lack of awareness (49\%) and fear of side effects (18\%) are the most common reasons for non-immunization with the influenza vaccine [17]. Malhi et al found that in Canada the rate of self-reported vaccinations among IBD patients is significantly low. The vaccination rates were reported as influenza $61.3 \%$, pneumococcus $10.3 \%$, HBV $61.0 \%$, hepatitis A virus $52.0 \%$, varicella $26.0 \%$, meningococcus $20.7 \%$, herpes 
Table 3 Screening test and recommendation for patients with inflammatory bowel disease [99-101]

\begin{tabular}{llll}
\hline Infection & Test & Recommendation for screening & Vaccine \\
\hline TB & $\begin{array}{l}\text { QuantiFERON TB-Gold } \\
\text { and tuberculin skin test }\end{array}$ & Yes & $\begin{array}{l}\text { Contraindication in } \\
\text { immunocompromised }\end{array}$ \\
Hepatitis B virus & HBsAg and HBsAb & Yes & Yes \\
HCV & Anti-HCV serology & Yes & No \\
HIV & HIV serology & Yes & No \\
Varicella zoster virus & VZV serology & Yes & Yes \\
Human papilloma virus & Cervical cytology & Yes & Yes \\
\hline
\end{tabular}

TB, tuberculosis; HCV, hepatitis C virus; HIV, human immunodeficiency virus; VZV, varicella zoster virus

zoster (HZ) 5.3\%, and human papillomavirus (HPV) $11.0 \%$. Among IBD patients, insufficient counseling by providers, ambiguity about indications and fears concerning vaccine safety are the most common reasons for non-uptake $(22.0 \%$, $20.7 \%$ and $5.3 \%$, respectively) [7]. Additionally, physician uncertainties over whether vaccination is indicated in IBD patients and a lack of knowledge about immunizations on the part of providers have been reported $[18,19]$. Regrettably, data showed only $30 \%$ of family medicine specialists felt comfortable managing routine maintenance issues including immunization in the IBD patients, especially when they were immunocompromised $[8,20]$.

\section{Vaccination recommendations}

Current practice recommendations proposed by the second European evidence-based consensus for routine vaccinations in IBD patients are presented in (Table 4) [21].

\section{Influenza}

All patients diagnosed with IBD should be immunized with the influenza vaccine yearly [22]. There are two forms of influenza vaccines: an inactivated form injected intramuscularly and intradermally, and a live form administered intranasally [3]. The inactivated influenza vaccine is safe to be given to patients on immunomodulators or biologic therapy. However, the live intranasal vaccination should be avoided in patients who are immunosuppressed [23].

DeBruyn et al showed in a randomized study that, in 137 patients with IBD, serologic protection against the influenza vaccine was reached by around $45-80 \%$ on maintenance infliximab therapy, varying by antigen. Essentially, vaccine timing relative to infliximab infusion did not affect the attainment of serologic protection and the influenza vaccine was well tolerated. Consequently, vaccination against influenza is recommended at any point throughout infliximab scheduling [24]. Cullen et al reported that, among 108 IBD patients taking the $2009 \mathrm{H} 1 \mathrm{~N} 1$ influenza vaccine, the proportion with seroprotection was considerably lower among individuals on combination immunosuppression therapy compared to those not treated with immunosuppressive medications (36\% vs. $64 \%, \mathrm{P}=0.02$ ) [25]. Additionally, Hiroko et al, in a prospective randomized controlled trial, found that booster doses of the trivalent influenza vaccine were not able to induce a significant immune response in adult IBD patients [26]. Importantly, data showed that the higher dose of influenza vaccine in persons 65 years of age or older triggered significantly higher antibody responses and offered better protection against laboratoryconfirmed influenza disease [25].

\section{Pneumococcal infection}

Streptococcus pneumoniae is a pathological microorganism that can cause severe infections, such as pneumonia or meningitis [27]. A study in Denmark reported that, even before the diagnosis of IBD, this group of patients was prone to be infected with pneumococcal pneumonia, signifying that the existence of IBD increases the chance of infection $[28,29]$. In a retrospective cohort study performed among IBD patients who matched non-IBD individuals, the IBD group had a higher risk of developing pneumonia than did patients without IBD (incidence rate ratio [IRR] 1.82, 95\%CI 1.75-1.88). It was shown that the use of biologic medications (OR 1.28, 95\% CI1.08-1.52), steroids (OR 3.62, 95\%CI 3.30-3.98) or proton pump inhibitors (OR 1.14, 95\%CI 1.03-1.25) within 120 days was strongly related to pneumonia [30]. Therefore, the immunization status should be updated even before the initiation of immunosuppressive therapy [28,31].

Presently, there are two forms of pneumococcal vaccines available: 23-valent polysaccharide vaccine (PPSV23) and 13-valent conjugate vaccine (PCV13). According to a recommendation of the Advisory Committee on Immunization Practices (ACIP), all IBD patients should be vaccinated with both PCV13 and PPSV23. A single dose of PCV13 should be given to all IBD patients, followed by a dose of PPSV23 at least 8 weeks later in immunodeficient patients, or after 1 year in immunocompetent patients. A second dose of PPSV23 should be given 5 years after the first dose and needs to be repeated in adults aged 65 years or older. If IBD patients previously received the PPSV23 vaccine, a single dose of the PVC13 vaccine should be administered at least 1 year later, regardless of the patient's immune status $[32,33]$. The recommendations of the European consensus on opportunistic infection in IBD patients are parallel to those in the USA, where all patients with 
Table 4 Summary of current practice recommendations for routine vaccinations in patients with inflammatory bowel disease [21]

Vaccines recommended per routine guidelines

\begin{tabular}{l}
\hline Tetanus (as part of Td, Tdap, or DTaP) \\
HPV (quadrivalent vaccine against types $6,11,16$, and 18) \\
Hepatitis A (single-antigen vaccine or as part of hepatitis A and B combination vaccine) \\
Hepatitis B vaccine \\
Poliomyelitis \\
Pertussis (as part of Tdap or DTaP) \\
Inactivated influenza (trivalent inactivated vaccine, annually) \\
\hline
\end{tabular}

Vaccines recommended prior to initiation of immune-modulator therapy

Varicella vaccine $^{b}$

Pneumococcus vaccine (PCV13, PPSV23)

${ }^{\mathrm{a} B o t h}$ males and females, according to national guidelines. The current Canadian guidelines recommend the HPV vaccine to both males and females between 9 and 26 years of age

${ }^{\mathrm{b}}$ In those without a clear history of chickenpox, shingles, or receipt of two doses of varicella vaccine and seronegative for varicella zoster virus antibody [VZV] IgG

'The specific sequence of administration for these two vaccines varies with patient characteristics and history of prior vaccination, as outlined in the CDC-ACIP vaccination schedule [http://www.cdc.gov/vaccines/schedules/downloads/adult/adult-schedule.pdf]

Td, tetanus, diphtheria; Tdap, tetanus, diphtheria, and acellular pertussis; DTaP, pediatric combination vaccine against tetanus, diphtheria, and acellular pertussis; HPV, human papillomavirus; PCV13, 13-valent pneumococcal conjugate vaccine; PPSV23, 23-valent pneumococcal polysaccharide vaccine; CDC-ACIP, Centers for Disease Control and Prevention - Advisory Committee on Immunization Practices

IBD treated with immunosuppressive medications, and who have not previously been vaccinated with PCV13 or PPV23, should be given a single dose of PCV13, followed by a dose of PPV23 at least 8 weeks later. A second dose of PPV23 vaccine is suggested 5 years later. Patients who have previously received PPV23 should be vaccinated with a PCV13 dose 12 months after their last PPV23 [21].

\section{HZ}

Varicella zoster virus (VZV) is a worldwide pathogen that only infects humans. Primary infection by VZV leads to varicella (chickenpox) disorder and subsequently the virus remains dormant in the nervous system. Treatment with immunosuppressive agents can debilitate cell-medicated immunity to VZV, leading to its reactivation [34]. VZV infection risk is high for IBD patients $[35,36]$. A retrospective cohort study by Long et al showed that the IBD cohort had a higher risk of $\mathrm{HZ}$ infection compared with the general population (IRR 1.68, 95\%CI 1.60-1.76) [37]. Moreover, in another study by Gupta et al, patients with ulcerative colitis and Crohn's disease had a higher incidence of zoster infection compared with their matched controls. The investigators reported that the use of azathioprine/6mercaptopurine medications (adjusted OR 3.1, 95\%CI 1.7-5.6) in corticosteroid recipients (adjusted OR 1.5, 95\%CI 1.1-2.2) was associated with a higher chance of developing shingles [38]. In line with previous studies, Cullen et al showed that VZV can be associated with a significant risk of morbidity and mortality in immunosuppressed patients, especially those under treatment with corticosteroids and combination immunosuppression, including methotrexate and azathioprine [39].
Immunosuppression increases the risk, but not all immunosuppression might carry the same risk, as vedolizumab has been reported to have a low incidence of serious infections [40]. Moreover, another study by Papp et al demonstrated that the rates of serious infection for infliximab and other biologics were significantly greater than that for ustekinumab [41].

The HZ vaccine is recommended in IBD patients aged 60 years and older, regardless of whether they have had a previous zoster episode. The vaccination is effective for reducing the incidence of $\mathrm{HZ}$ by $51 \%$ and post-herpetic neuralgia by $67 \%$ [42]. However, the zoster vaccine is contraindicated while IBD patients are on biological agents, given the fact that the zoster vaccine is a live, attenuated vaccine. Given the risk of developing $\mathrm{HZ}$ infections, this vaccine should be considered even in those managed with low-dose immunosuppression, such as low-dose prednisone ( $<20 \mathrm{mg}$ /daily), 6-mercaptopurine $(<1.5 \mathrm{mg} / \mathrm{kg} /$ day $)$ or azathioprine $(<2.5 \mathrm{mg} / \mathrm{kg} /$ day $)$ [43]. Therefore, the vaccine needs to be given $\geq 3$ weeks prior to the initiation of any immunosuppressant medication [44]. However, Khan et al showed that, in 59 patients treated with anti-TNF medication, of whom $12(20 \%)$ were also using thiopurine, once they received the vaccine no case of $\mathrm{HZ}$ infection was seen within 0-42 days after its administration [45].

\section{Varicella}

It was suggested by the Centers for Disease Control and Prevention (CDC) that all individuals who lack evidence of VZV immunity by serology testing should receive two doses of varicella vaccine, given at least 4-8 weeks apart [46]. All IBD patients need to be screened for immunity to VZV at 
the time of diagnosis. Unimmunized and immunocompetent IBD patients should be offered vaccination with a 2-dose series of live varicella vaccine at least 3 weeks prior to the start of immunosuppressive therapy. Because varicella vaccine is a live virus vaccine, it should be administered in immunocompromised patients at least 3 months after the immunosuppressive treatment is discontinued [4,47]. In line with this, the European Crohn's and Colitis Organisation suggests administering the live vaccine either 3 weeks prior to starting treatment or 3-6 months after discontinuation of immunosuppressive agents [21,48]. Lindsey et al showed that the $\mathrm{HZ}$ vaccination (Zostavax) was safe in patients with rheumatoid, psoriatic arthritis and spondyloarthropathies who were on infused biologics [49].

Because the inactivated zoster vaccine $\left(\mathrm{ZV}_{\mathrm{IN}}\right)$ is not a live vaccine, no issues should be expected when it is administered to immunocompromised patients. Parrino et al showed that, in patients with hematologic malignancies receiving antiCD20 monoclonal antibodies, $\mathrm{ZV}_{\mathrm{IN}}$ was tolerated well and caused statistically significant VZV-specific T-cell immune responses [50]. Additionally, another study reported that, in patients with autoimmune diseases, $\mathrm{ZV}_{\mathrm{IN}}$ produced statistically significant immune responses [51].

The presence of VZV antibodies shows a prior infection with varicella and protection against this virus. VZV-specific antibody testing to measure immunity in those previously immunized with the varicella vaccine is not indorsed by the Advisory Committee on Immunization Practices [52]. As data showed, the reason for this is that the varicella vaccine produces lower VZV antigen-antibody concentration compared to natural immunity after varicella infection.

The recent commercial antibody, enzyme-linked immunosorbent assay (ELISA), is not sensitive enough to assess vaccine-induced VZV antibody levels in all patients, particularly those with a distant history of vaccination. Investigators from the CDC discovered that their ELISA, comparable with or more sensitive than commercial assays, had a $34 \%$ false-negative rate when compared with the glycoprotein ELISA developed by Merck [52,53].

Gastroenterologists need to be aware of the limitations of VZV serology using the commercially available immunoassays when they measure varicella immunity in those immunized. We need to rely more upon the patient's history of immunization, rather than current commercial ELISA, to evaluate the immunity to varicella [54].

\section{Tetanus, diphtheria, acellular pertussis}

Diphtheria and tetanus have become uncommon infections in developed countries but outbreaks have occurred in former Soviet Republics [55]. As per ACIP recommendations, adults who did not receive primary vaccination, or who did not complete the primary series, should begin or complete the primary vaccination series with three doses of tetanus- and diphtheria-containing vaccines, one of which should be a Tdap dose. All IBD patients should be given the combined tetanus and diphtheria toxoids (Td) booster every 10 years, regardless of their immunosuppression status [56]. In 2014, 40,727 cases of pertussis were reported to The European Surveillance System (TESSy) by 29 countries of the European Union (EU) or European Economic Area (EEA) [57]. Additionally, in 2015, state health departments reported 20,762 cases of pertussis to the CDC $[58,56]$. Regardless of previous Tdap vaccination records, Tdap was recommended by ACIP for all pregnant women in the third trimester of their pregnancies [59]. The Tdap vaccine was recommended for all pregnant women in the United Kingdom and Ireland (Table 5) [60,61]. Dezfoli et al, in a controlled trial, evaluated the immunogenicity of the Tdap vaccine and found that, regardless of immunosuppressive regimen, patients have a normal booster response. They suggested patients with IBD should be vaccinated with Tdap prior to starting immunomodulators, especially once combination therapy with anti-TNF is started [62]. Additionally, in a cross-sectional study, Caldera et al showed that IBD patients on combination therapy or biological monotherapy had lower sustained pertussis antibody concentration [63]. Brogan et al suggested that patients with IBD had significantly impaired in vitro production of anti-tetanus toxoid antibody during an 8-day pokeweed mitogen-stimulated culture period. They reported that their results indicated many IBD patients have an impaired humoral immune response to tetanus toxoid booster immunization. This impaired immune response may be due to an inability to generate $B$ cell precursors of antitetanus toxoid IgG-producing B cells, rather than to abnormal circulating helper or suppressor T-cell activity or natural killer cell regulatory activity [64].

\section{Measles, mumps, rubella (MMR)}

According to The Regional Verification Commission for Measles and Rubella Elimination at the WHO Regional Office for Europe, measles elimination was not reached in 14 of the 53 member states (26\%) of the WHO European Region at the end of 2015 [65]. In January-February 2017, 10 EU/EEA countries reported more than double the number of cases compared to the same period in 2016 [66].

The effectiveness of the measles-component of the MMR vaccine was reported as $95-98 \%$ after a single dose and more than $99 \%$ following the second dose of the vaccine [67]. Similarly to measles protection, the MMR vaccine achieves more than 95\% seroprotection against rubella after 1 dose and more than $99 \%$ following 2 doses $[67,68]$. However, the potency of the MMR vaccine against mumps is not as effective as against measles and rubella: seroconversion for mumps is $64-95 \%$ with 1 dose of vaccine and $88-95 \%$ following 2 doses $[67,68]$.

Cleveland et al, in a prospective study, showed that a significant number of IBD patients lack immunity to measles [69]. The MMR vaccine is only available as a live, attenuated vaccine. Therefore, immunocompromised IBD patients should not be vaccinated with MMR [70]. Consequently, this vaccine should be administered at least 
Table 5 Vaccination guidelines against tetanus, diphtheria, and pertussis [60-61]

\begin{tabular}{|c|c|c|}
\hline Country & Adult (18-65 years) & Elderly $>65$ years \\
\hline Austria $^{\mathrm{a}}$ & Every 10 years & Every 5 years \\
\hline Belgium $^{b}$ & Every 10 years & Every 10 years \\
\hline Cyprus & Every 10 years & Every 10 years \\
\hline Denmark & - & - \\
\hline Sweden & Every 20 years & Every 20 years \\
\hline France & ${ }^{c}$ Td-IPV at age 25 and 45 years & Every 10 years \\
\hline Germany & Every 10 years & Every 10 years \\
\hline Ireland & Tdap for each pregnant women & - \\
\hline Norway & - & - \\
\hline United Kingdom & Tdap for each pregnant women & - \\
\hline Spain & - & $\mathrm{Td}$ at age around 65 years \\
\hline Italy & Every 10 years & - \\
\hline \multicolumn{3}{|c|}{$\begin{array}{l}\text { Diphtheria, tetanus, acellular pertussis (DTaP) and inactivated poliomyelitis vaccine (DTaP-IPV) should be given every } 10 \text { years between } 18 \text { and } 60 \text { years of } \\
\text { age }\end{array}$} \\
\hline \multicolumn{3}{|c|}{${ }^{b}$ One of the booster doses should contain the pertussis antigen (Tdap) for those who have not previously received a single dose of Tdap } \\
\hline cFor those who did & $\begin{array}{l}\text { g vaccine during the past } 5 \text { year } \\
25\end{array}$ & he (DTaP-IPV) \\
\hline
\end{tabular}

1 month before the start of immunosuppressive agents [71]. The MMR vaccine can be given safely to the household or close contacts of an immunocompromised IBD patient [67].

\section{HPV}

The International Agency for Research on Cancer (IARC) has shown proof of a strong association between HPV and cancer sites such as anus and cervix [72]. HPV might be accountable for other malignancies, including cancer of the esophagus, oral cavity and lip. However, a causal role for HPV has not been recognized [73]. The incidence of HPVrelated cancers is typically higher in immunosuppressed patients [74]. Shah et al demonstrated that there was a trend toward abnormal anal Papanicolaou in IBD subjects compared with a healthy control. There was no difference based on immunosuppression [75].

The quadrivalent HPV (qHPV) vaccine has been proven to prevent vaccine-related persistent anal HPV infections in addition to anal intraepithelial neoplasia [76]. A handful of studies have examined the prevalence of cervical dysplasia in IBD patients. Jacobson et al reported that IBD patients aged 9-26 on immunosuppressive therapy showed a proper immune response with $100 \%$ seroconversion to the HPV4 quadrivalent vaccine (against HPV types 16, 18, 6, and 11). They did not find any serious side-effects or worsening of disease activity due to the HPV4 vaccine [77]. Allegretti at al showed that IBD patients on immunosuppressive medication have a higher risk of high-grade cervical dysplasia and cervical cancer (OR 1.34, 95\%CI 1.23-1.46) compared with the general population [78]. Likewise, Singh et al documented that combination therapy with steroids and immunosuppressive agents can increase the risk of cervical abnormalities (OR 1.46, 95\%CI 1.09-1.81) compared with IBD patients who are not on these medications [79]. The recommendation for the HPV vaccination schedules was updated by the CDC in October 2016. It was advised 2 doses of HPV vaccine for individuals younger than 15 years and 3 doses of HPV vaccine series for those aged 15 or older and have certain immunodeficiency conditions. The CDC continues to recommend routine HPV vaccination series for girls and boys aged 11 or 12 years. For immunocompromised patients aged 9 to 26 years, 3 doses of HPV vaccine (0, 1-2, 6 months) are recommended. The HPV vaccination series can be started at age 9 years and is also suggested for women through age 26 years and men through age 21 years. Individuals whose immune responses might be insufficient or lower (because of HIV infection, malignancy, autoimmune disorder, or use of immunosuppressant medications) should receive 3 doses to ensure they receive the most benefit [80]. In Europe there are two vaccines (Cervarix@ and Gardasil@) against HPV authorized centrally by the European Medicines Agency. Cervarix is itemized to be administered with the reduced schedule in girls aged 9-14 years and Gardasil received positive feedback for use in 9-13-year-old adolescent girls and boys [81].

\section{IBD vaccination and travel}

All IBD patients who plan to travel overseas need to check what specific vaccinations they need according to where they are planning to travel [82]. It is important that they discuss their travel plans with a traveler's clinic beforehand and to go over the required vaccinations. They should familiarize themselves with the endemic infections of the specific areas 
they plan to visit by reviewing the traveler's health information from the World Health Organization [83]. Yellow fever is one of the biggest challenges for IBD patients who plan to visit South America and sub-Saharan Africa. Unfortunately, the yellow fever vaccine is a live vaccine and should be avoided in drug-induced immunocompromised IBD patients [82]. The yellow fever vaccine can be given to this group of patients if immunosuppressive medications are discontinued for at least 4 months before vaccination $[23,84]$. Otherwise, if their immunosuppressive medications cannot be terminated because of medical necessity, they need to be instructed against visiting areas where yellow fever is endemic [23]. Enteric fever is another serious disease that IBD patients should be concerned about if they plan to travel to the Indian subcontinent. Accordingly, all patients with IBD need to be vaccinated with the parenteral inactive typhoid vaccine (Vi vaccine) before they travel [85]. Rabies is a fatal disease that is broadly distributed throughout the world and cell-culture-derived vaccines are available for use by IBD patients traveling to high risk areas such as Africa, Asia and Latin America [86,87]. Regarding HBV, the immune status needs to be screened if there is intention to visit areas where $\mathrm{HBV}$ is endemic, such as Africa, China and Southeast Asia. HBV booster should be offered to those who are immunocompromised and whose immune titers are less than $10 \mathrm{mIU} / \mathrm{mL}$ [88].

Viral meningoencephalitis is mostly caused by Japanese encephalitis (JE) in large parts of Asia [89]. Patients on antiTNF therapy or dealing with a chronic medical condition may qualify for the JE vaccine [18]. An inactivated Japanese encephalitis vaccine (IXIARO ${ }^{\circ}$ ) has been approved in Europe and the United States and can be safely offered to IBD patients $[82,90,91]$.

\section{Concluding remarks}

Proper immunizations are an essential part of medical management in IBD patients [20]. Because immunomodulators are used to treat IBD patients, these patients are susceptible to infection, with a high rate of morbidity and mortality [92,93]. Therefore, this group of patients should be immunized prophylactically against these infections [94], preferably upon initial presentation and once the start of immunosuppressive agents is planned [95]. Given the increased risk of vaccinerelated infections, live vaccines should be avoided in immunocompromised patients. Importantly, the majority of immunocompromised patients exhibit a proper and sufficient seroconversion once they are vaccinated [3].

However, vaccination rates for these preventable diseases continue to be suboptimal in the face of a decade of research proving that IBD patients are at an increased risk of vaccinepreventable infections [96]. Prior data have shown that recommendations from physicians are the most important factor for receiving preventative health services such as vaccination and screening for cancer [97]. As per previous data, in the majority of IBD patients the screening test for HBV serology was missed by their gastroenterologists. This suggests that providers may not be effectively instructed and do not regularly recommend screening for HBV and vaccination for their IBD patients, whether they are on or off immunosuppressive medications [98]. Vaccination assessments yearly, and prior to initiation of treatment with immunosuppressive agents, were important predictors of vaccination completion [7].

In conclusion, taking care of patients with IBD often includes making complex medical decisions. Gastroenterologists are usually the primary providers for patients with IBD; consequently, it is critical to have a broad knowledge of the issues surrounding the administration of vaccines to patients with IBD. The vaccination recommendation should be tailored to each patient, taking into account his/her age, comorbidities, nutritional status, IBD disease severity, immunosuppressive therapy, risk of exposure to pathogens and geographic clustering. Moreover, vaccination should not delay urgent medical therapy and gastroenterologists should involve infectious specialists when they face a challenging situation not addressed by guidelines.

\section{References}

1. Rubin DC, Shaker A, Levin MS. Chronic intestinal inflammation: inflammatory bowel disease and colitis-associated colon cancer. Front Immunol 2012;3:107.

2. Bernheim O, Colombel JF, Ullman TA, Laharie D, Beaugerie L, Itzkowitz SH. The management of immunosuppression in patients with inflammatory bowel disease and cancer. Gut 2013;62:15231528.

3. Chaudrey K, Salvaggio M, Ahmed A, Mahmood S, Ali T. Updates in vaccination: recommendations for adult inflammatory bowel disease patients. World J Gastroenterol 2015;21:3184-3196.

4. Sands BE, Cuffari C, Katz J, et al. Guidelines for immunizations in patients with inflammatory bowel disease. Inflamm Bowel Dis 2004;10:677-692.

5. Nguyen DL, Nguyen ET, Bechtold ML. Effect of immunosuppressive therapies for the treatment of inflammatory bowel disease on response to routine vaccinations: a metaanalysis. Dig Dis Sci 2015;60:2446-2453.

6. Melmed GY. Vaccination strategies for patients with inflammatory bowel disease on immunomodulators and biologics. Inflamm Bowel Dis 2009;15:1410-1416.

7. Malhi G, Rumman A, Thanabalan R, et al. Vaccination in inflammatory bowel disease patients: attitudes, knowledge, and uptake. J Crohns Colitis 2015;9:439-444.

8. Selby L, Hoellein A, Wilson JF. Are primary care providers uncomfortable providing routine preventive care for inflammatory bowel disease patients? Dig Dis Sci 2011;56:819-824.

9. Sánchez-Tembleque MD, Corella C, Pérez-Calle JL. Vaccines and recommendations for their use in inflammatory bowel disease. World J Gastroenterol 2013;19:1354-1358.

10. Rubin LG, Levin MJ, Ljungman P, et al. 2013 IDSA clinical practice guideline for vaccination of the immunocompromised host. Clin Infect Dis 2014;58:e44-e100.

11. Andrisani G, Armuzzi A, Marzo M, et al. What is the best way to manage screening for infections and vaccination of inflammatory bowel disease patients? World J Gastrointest Pharmacol Ther 2016;7:387-396.

12. Kirchgesner J, Lemaitre M, Carrat F, Zureik M, Carbonnel F, Dray- 
Spira R. Risk of serious and opportunistic infections associated with treatment of inflammatory bowel diseases. Gastroenterology 2018;155:337-346.

13. Liu XQ, Qin L. Treatment strategy of inflammatory bowel disease associated with viral hepatitis. Chin J Gastroenterol Hepatol 2016;25:1091-1093.

14. Huang ML, Xu XT, Shen J, Qiao YQ, Dai ZH, Ran ZH. Prevalence and factors related to hepatitis $\mathrm{B}$ and $\mathrm{C}$ infection in inflammatory bowel disease patients in China: a retrospective study. J Crohns Colitis 2014;8:282-287.

15. Papa A, Felice C, Marzo M, et al. Prevalence and natural history of hepatitis $\mathrm{B}$ and $\mathrm{C}$ infections in a large population of IBD patients treated with anti-tumor necrosis factor- $\alpha$ agents. J Crohns Colitis 2013;7:113-119.

16. Jauregui-Amezaga A, Turon F, Ordás I, et al. Risk of developing tuberculosis under anti-TNF treatment despite latent infection screening. J Crohns Colitis 2013;7:208-212.

17. Melmed GY, Ippoliti AF, Papadakis KA, et al. Patients with inflammatory bowel disease are at risk for vaccine-preventable illnesses. Am J Gastroenterol 2006;101:1834-1840.

18. Gupta A, Macrae FA, Gibson PR. Vaccination and screening for infections in patients with inflammatory bowel disease: a survey of Australian gastroenterologists. Intern Med J 2011;41:462-467.

19. Jung YS, Park JH, Kim HJ, et al. Insufficient knowledge of Korean gastroenterologists regarding the vaccination of patients with inflammatory bowel disease. Gut Liver 2014;8:242-247.

20. Wasan SK, Calderwood AH, Long MD, Kappelman MD, Sandler RS, Farraye FA. Immunization rates and vaccine beliefs among patients with inflammatory bowel disease: an opportunity for improvement. Inflamm Bowel Dis 2014;20:246-250.

21. Rahier JF, Magro F, Abreu C, et al; European Crohn's and Colitis Organisation (ECCO). Second European evidence-based consensus on the prevention, diagnosis and management of opportunistic infections in inflammatory bowel disease. J Crohns Colitis 2014;8:443-468.

22. Harmandar F, Çekin AH. Preventive care in inflammatory bowel disease. Turk J Gastroenterol 2017;28:307-310.

23. Reich J, Wasan S, Farraye FA. Vaccinating patients with inflammatory bowel disease. Gastroenterol Hepatol (N Y) 2016;12:540-546.

24. deBruyn J, Fonseca K, Ghosh S, et al. Immunogenicity of influenza vaccine for patients with inflammatory bowel disease on maintenance Infliximab therapy: a randomized trial. Inflamm Bowel Dis 2016;22:638-647.

25. Cullen G, Bader C, Korzenik JR, Sands BE. Serological response to the $2009 \mathrm{H} 1 \mathrm{~N} 1$ influenza vaccination in patients with inflammatory bowel disease. Gut 2012;61:385-391.

26. Hiroko M, Watanabe $\mathrm{K}$, Morimoto $\mathrm{K}$, et al. Booster doses of the trivalent influenza vaccine do not elicit a significant immune response in patients with inflammatory bowel disease: a prospective randomized controlled trial. Gastroenterology 2014;146 Suppl 1:S-80.

27. Marín AC, Gisbert JP, Chaparro M. Immunogenicity and mechanisms impairing the response to vaccines in inflammatory bowel disease. World J Gastroenterol 2015;21:11273-11281.

28. Farraye FA. Vaccination of patients with inflammatory bowel disease. Gastroenterol Hepatol (N Y) 2017;13:431-434.

29. Kantsø B, Simonsen J, Hoffmann S, Valentiner-Branth P, Petersen AM, Jess T. Inflammatory bowel disease patients are at increased risk of invasive pneumococcal disease: a Nationwide Danish Cohort Study 1977-2013. Am J Gastroenterol 2015;110:1582-1587.

30. Long MD, Martin C, Sandler RS, Kappelman MD. Increased risk of pneumonia among patients with inflammatory bowel disease. Am J Gastroenterol 2013;108:240-248.
31. Lu Y, Bousvaros A. Immunizations in children with inflammatory bowel disease treated with immunosuppressive therapy. Gastroenterol Hepatol (N Y) 2014;10:355-363.

32. Centers for Disease Control and Prevention (CDC). Use of 13 -valent pneumococcal conjugate vaccine and 23-valent pneumococcal polysaccharide vaccine for adults with immunocompromising conditions: recommendations of the Advisory Committee on Immunization Practices (ACIP). MMWR Morb Mortal Wkly Rep 2012;61:816-819.

33. Centers for Disease Control and Prevention. Use of 13-valent pneumococcal conjugate vaccine and 23-valent pneumococcal polysaccharide vaccine among children aged 6-18 years with immunocompromising conditions: recommendations of the Advisory Committee on Immunization Practices (ACIP). MMWR Morb Mortal Wkly Rep 2015;62:521-524.

34. Nagel MA, Gilden D. Complications of varicella zoster virus reactivation. Curr Treat Options Neurol 2013;15:439-453.

35. Fidder H, Schnitzler F, Ferrante M, et al. Long-term safety of infliximab for the treatment of inflammatory bowel disease: a single-centre cohort study. Gut 2009;58:501-508.

36. Ma C, Walters B, Fedorak RN. Varicella zoster meningitis complicating combined anti-tumor necrosis factor and corticosteroid therapy in Crohn's disease. World J Gastroenterol 2013; 19:3347-3351.

37. Long MD, Martin C, Sandler RS, Kappelman MD. Increased risk of herpes zoster among 108604 patients with inflammatory bowel disease. Aliment Pharmacol Ther 2013;37:420-429.

38. Gupta G, Lautenbach E, Lewis JD. Incidence and risk factors for herpes zoster among patients with inflammatory bowel disease. Clin Gastroenterol Hepatol 2006;4:1483-1490.

39. Cullen G, Baden RP, Cheifetz AS. Varicella zoster virus infection in inflammatory bowel disease. Inflamm Bowel Dis 2012;18:23922403.

40. Colombel JF, Sands BE, Rutgeerts P, et al. The safety of vedolizumab for ulcerative colitis and Crohn's disease. Gut 2017;66:839-851.

41. Papp K, Gottlieb AB, Naldi L, et al. Safety surveillance for ustekinumab and other psoriasis treatments from the psoriasis longitudinal assessment and registry (PSOLAR). J Drugs Dermatol 2015;14:706-714.

42. Oxman MN, Levin MJ, Johnson GR, et al; Shingles Prevention Study Group. A vaccine to prevent herpes zoster and postherpetic neuralgia in older adults. N Engl J Med 2005;352:2271-2284.

43. Harpaz R, Ortega-Sanchez IR, Seward JF; Advisory Committee on Immunization Practices (ACIP) Centers for Disease Control and Prevention (CDC). Prevention of herpes zoster: recommendations of the Advisory Committee on Immunization Practices (ACIP). MMWR Recomm Rep 2008;57:1-30.

44. Ali T, Kaitha S, Mahmood S, Ftesi A, Stone J, Bronze MS. Clinical use of anti-TNF therapy and increased risk of infections. Drug Healthc Patient Saf 2013;5:79-99.

45. Khan N, Shah Y, Trivedi C, Lewis JD. Safety of herpes zoster vaccination among inflammatory bowel disease patients being treated with anti-TNF medications. Aliment Pharmacol Ther 2017;46:668-672.

46. Kim DK, Riley LE, Hunter P. Advisory committee on immunization practices recommended immunization schedule for adults aged 19 years or older - United States. MMWR Morb Mortal Wkly Rep 2018;67:158-160.

47. Rahier JF, Moutschen M, Van Gompel A, et al. Vaccinations in patients with immune-mediated inflammatory diseases. Rheumatology (Oxford) 2010;49:1815-1827.

48. Côté-Daigneault J, Peerani F, MacMahon E, Delaporte E, Rahier JF, Colombel JF. Management and prevention of Herpes Zoster in the immunocompromised inflammatory bowel disease patient: a clinical quandary. Inflamm Bowel Dis 2016;22:2538- 
2547.

49. Lindsey S, Oufnac B, Walker H. Safety of Zoster vaccination administration in rheumatic patients on current biologic therapy. ACR/ARHP Annual Meeting 2014, Abstract No. 1836.

50. Parrino J, McNeil SA, Lawrence SJ, et al. Safety and immunogenicity of inactivated varicella-zoster virus vaccine in adults with hematologic malignancies receiving treatment with anti-CD20 monoclonal antibodies. Vaccine 2017;35:1764-1769.

51. Eberhardson M, Hall S, Papp KA, et al. Safety and immunogenicity of inactivated Varicella-Zoster virus vaccine in adults with autoimmune disease: a phase 2, randomized, double-blind, placebo-controlled clinical trial. Clin Infect Dis 2017;65:11741182.

52. Marin M, Güris D, Chaves SS, Schmid S, Seward JF; Advisory Committee on Immunization Practices, Centers for Disease Control and Prevention (CDC). Prevention of varicella. Recommendations of the advisory committee on immunization practices. MMWR Morb Mortal Wkly Rep 2007;56(RR-4):1-40.

53. Caldera F, Wasan SK., Farraye FA, Hayney MS. Caution when assessing immunity to varicella through antibody testing in patients with inflammatory bowel disease. Inflamm Bowel Dis 2017;23:E50-E51.

54. Liu J, Ye X, Jia J, et al. Serological evaluation of immunity to the varicella-zoster virus based on a novel competitive enzyme-linked immunosorbent assay. Sci Rep 2016;6:20577.

55. Golaz A, Hardy IR, Strebel P, et al. Epidemic diphtheria in the newly independent states of the former Soviet Union: implications for diphtheria control in the United States. J Infect Dis 2000;181(Suppl 1):S237-S243.

56. Kim DK, Bridges CB, Harriman KH; Advisory Committee on Immunization Practices (ACIP): Advisory Committee on Immunization Practices Recommended Immunization schedule for adults aged 19 years or older - United States, 2016. MMWR Recomm Rep 2016;65:88-90.

57. European Centre for Disease Prevention and Control. Annual Epidemiological Report 2016 - Pertussis. 2016. https://ecdc. europa.eu/sites/portal/files/documents/AER-for-2016-pertussis. pdf [Accessed January 1, 2019].

58. Centers for Disease Control and Prevention (CDC). Pertussis (whooping cough): surveillance and reporting. https://www.cdc. gov/pertussis/outbreaks/trends.html [Accessed January 1, 2019]

59. Centers for Disease Control and Prevention (CDC). Updated recommendations for use of tetanus toxoid, reduced diphtheria toxoid, and acellular pertussis vaccine (Tdap) in pregnant women-Advisory Committee on Immunization Practices (ACIP), 2012. MMWR Morb Mortal Wkly Rep 2013;62:131-135.

60. World Health Organization. WHO vaccine-preventable diseases: monitoring system: 2016 global summary. Geneva: World Health Organization, 2016. http://apps.who.int/immunization monitoring/globalsummary [Accessed January 1, 2019].

61. European Centre for Disease Prevention and Control Vaccine. European Centre for Disease Prevention and Control; 2016. http://vaccine-schedule.ecdc.europa.eu/Pages/Scheduler.aspx [Accessed January 1, 2019].

62. Dezfoli S, Horton HA, Thepyasuwan N, et al. Combined immunosuppression impairs immunogenicity to tetanus and pertussis vaccination among patients with inflammatory bowel disease. Inflamm Bowel Dis 2015;21:1754-1760.

63. Caldera F, Saha S, Wald A, et al. Lower sustained diphtheria and pertussis antibody concentrations in inflammatory bowel disease patients. Dig Dis Sci 2018;63:1532-1540.

64. Brogan MD, Shanahan F, Oliver M, Stevens RH, Targan SR. Defective memory B cell formation in patients with inflammatory bowel disease following tetanus toxoid booster immunization. J Clin Lab Immunol 1987;24:69-74.
65. World Health Organization (WHO) Regional Office for Europe. $5^{\text {th }}$ Meeting of the European Regional Verification Commission for Measles and Rubella Elimination (RVC). Copenhagen. WHO/ Europe; 2016. http://www.euro.who.int/_data/assets/pdf_ file/0005/330917/5th-RVC-meeting-report.pdf?ua=1 [Accessed January 1, 2019].

66. European Centre for Disease Prevention and Control (ECDC). Monthly measles epidemiological updates. Stockholm: ECDC. http://ecdc.europa.eu/en/healthtopics/measles/epidemiological_ data/Pages/measles_surveillance_reports.aspx [Accessed January 1,2019$]$.

67. Watson JC, Hadler SC, Dykewicz CA, Reef S, Phillips L. Measles, mumps, and rubella-vaccine use and strategies for elimination of measles, rubella, and congenital rubella syndrome and control of mumps: recommendations of the Advisory Committee on Immunization Practices (ACIP). MMWR Recomm Rep 1998;47:1-57.

68. White SJ, Boldt KL, Holditch SJ, Poland GA, Jacobson RM. Measles, mumps, and rubella. Clin Obstet Gynecol 2012;55: 550-559.

69. Cleveland NK, Rodriquez D, Wichman A, Pan I, Melmed GY, Rubin DT. Many inflammatory bowel disease patients are not immune to measles or pertussis. Dig Dis Sci 2016;61:2972-2976.

70. Kotton CN. Vaccines and inflammatory bowel disease. Dig Dis 2010;28:525-535.

71. Wasan SK, Baker SE, Skolnik PR, Farraye FA. A practical guide to vaccinating the inflammatory bowel disease patient. Am J Gastroenterol 2010;105:1231-1238.

72. IARC Working Group on the Evaluation of Carcinogenic Risks to Humans. Human papillomaviruses. IARC Monogr Eval Carcinog Risks Hum 2007;90:1-636.

73. Chaturvedi AK. Beyond cervical cancer: burden of other HPV-related cancers among men and women. J Adolesc Health 2010;46:S20-S26.

74. Bucchi D, Stracci F, Buonora N, Masanotti G. Human papillomavirus and gastrointestinal cancer: A review. World $J$ Gastroenterol 2016;22:7415-7430.

75. Shah SB, Pickham D, Araya H, et al. Prevalence of anal dysplasia in patients with inflammatory bowel disease. Clin Gastroenterol Hepatol 2015;13:1955-1961.e1.

76. Stier EA, Chigurupati NL, Fung L. Prophylactic HPV vaccination and anal cancer. Hum Vaccin Immunother 2016;12:1348-1351.

77. Jacobson DL, Bousvaros A, Ashworth L, et al. Immunogenicity and tolerability to human papillomavirus-like particle vaccine in girls and young women with inflammatory bowel disease. Inflamm Bowel Dis 2013;19:1441-1449.

78. Allegretti JR, Barnes EL, Cameron A. Are patients with inflammatory bowel disease on chronic immunosuppressive therapy at increased risk of cervical high-grade dysplasia/cancer? A meta-analysis. Inflamm Bowel Dis 2015;21:1089-1097.

79. Singh H, Demers AA, Nugent Z, Mahmud SM, Kliewer EV, Bernstein CN. Risk of cervical abnormalities in women with inflammatory bowel disease: a population-based nested casecontrol study. Gastroenterology 2009;136:451-458.

80. Walker TY, Elam-Evans LD, Singleton JA, et al. National, regional, state, and selected local area vaccination coverage among adolescents aged 13-17 years - United States, 2016. MMWR Morb Mortal Wkly Rep 2016;66:874-882.

81. https://www.ema.europa.eu/en/medicines/human/referrals/ human-papillomavirus-vaccines-cervarix-gardasil-gardasil-9silgard.

82. Esteve M, Loras C, García-Planella E. Inflammatory bowel disease in travelers: choosing the right vaccines and check-ups. World J Gastroenterol 2011;17:2708-2714.

83. International travel and health: traveller vaccinations. 
World Health Organization, 2016. http://www.who.int/ith/ updates/20110427/en/[Accessed January 1, 2019].

84. Ekenberg C, Friis-Møller N, Ulstrup T, Aalykke C. Inadvertent yellow fever vaccination of a patient with Crohn's disease treated with infliximab and methotrexate. BMJ Case Rep 2016:2016.

85. Whitaker JA, Franco-Paredes C, del Rio C, Edupuganti S. Rethinking typhoid fever vaccines: implications for travelers and people living in highly endemic areas. J Travel Med 2009;16:46-52.

86. Meslin FX. Rabies as a traveler's risk, especially in high-endemicity areas. J Travel Med 2005;12 Suppl 1:S30-S40.

87. Viget N, Vernier-Massouille G, Salmon-Ceron D, Yazdanpanah Y, Colombel JF. Opportunistic infections in patients with inflammatory bowel disease: prevention and diagnosis. Gut 2008;57:549-558.

88. Zanetti AR, Van Damme P, Shouval D. The global impact of vaccination against hepatitis $\mathrm{B}$ : a historical overview. Vaccine 2008;26:6266-6273.

89. Buhl MR, Lindquist L. Japanese encephalitis in travelers: review of cases and seasonal risk. J Travel Med 2009;16:217-219.

90. Burchard GD, Caumes E, Connor BA, et al. Expert opinion on vaccination of travelers against Japanese encephalitis. J Travel Med 2009;16:204-216.

91. Firbas C, Jilma B. Product review on the JE vaccine IXIARO. Hum Vaccin Immunother 2015;11:411-420.

92. Lichtenstein GR, Feagan BG, Cohen RD, et al. Serious infection and mortality in patients with Crohn's disease: more than 5 years of follow-up in the TREAT ${ }^{\text {mo }}$ registry. Am J Gastroenterol 2012;107:1409-1422.

93. Ananthakrishnan AN, McGinley EL. Infection-related hospitalizations are associated with increased mortality in patients with inflammatory bowel diseases. J Crohns Colitis 2013;7:107-112.

94. Narula N, Yamamura DL, Marshall JK. Should my patient with inflammatory bowel disease on immunosuppressive therapy be vaccinated against influenza virus? Can J Gastroenterol 2010;24:121-125.

95. Kim SB, Park SJ, Chung SH, et al. Vaccination and complementary and alternative medicine in patients with inflammatory bowel disease. Intest Res 2014;12:124-130.

96. Pham HV, Hasan I, Udaltsova N, et al. Rates and predictors of vaccinations among inflammatory bowel disease patients receiving anti-tumor necrosis factor agents. Dig Dis Sci 2018;63:209-217.
97. Blewett LA, Johnson PJ, Lee B, Scal PB. When a usual source of care and usual provider matter: adult prevention and screening services. J Gen Intern Med 2008;23:1354-1360.

98. Ben Musa R, Gampa A, Basu S, et al. Hepatitis B vaccination in patients with inflammatory bowel disease. World J Gastroenterol 2014;20:15358-15366.

99. Gandhi RT, Wurcel A, Lee H, et al. Isolated antibody to hepatitis $\mathrm{B}$ core antigen in human immunodeficiency virus type-1-infected individuals. Clin Infect Dis 2003;36:1602-1605.

100. Rahier JF, Ben-Horin S, Chowers Y, et al; European Crohn's and Colitis Organisation (ECCO). European evidence-based Consensus on the prevention, diagnosis and management of opportunistic infections in inflammatory bowel disease. J Crohns Colitis 2009;3:47-91.

101. Committee on Practice Bulletins-Gynecology. ACOG Practice Bulletin Number 131: Screening for cervical cancer. Obstet Gynecol 2012;120:1222-1238.

102. Centers for Disease Control and Prevention (CDC). Contraindications and precautions for polio vaccination. https://www.cdc.gov/vaccines/vpd/polio/hcp/contraindicationsprecautions.html [Accessed January 1, 2019].

103. Staples JE, Gershman M, Fischer M; Centers for Disease Control and Prevention (CDC). Yellow fever vaccine: recommendations of the Advisory Committee on Immunization Practices (ACIP). MMWR Recomm Rep 2010;59:1-27.

104. Staples JE, Bocchini JA Jr, Rubin L, Fischer M; Centers for Disease Control and Prevention. Yellow fever vaccine booster doses: recommendations of the advisory committee on immunization practices, 2015. MMWR Morb Mortal Wkly Rep 2015;64:647-650.

105. Petersen BW, Damon IK, Pertowski CA, et al. Clinical guidance for smallpox vaccine use in a postevent vaccination program. MMWR Recomm Rep 2015;64:1-26.

106. World Health Organization. Immunization, vaccines and biologicals: Contraindications. http://www.who.int/immunization/ policy/contraindications.pdf [Accessed January 1, 2019].

107. Jackson BR, Iqbal S, Mahon B; Centers for Disease Control and Prevention (CDC). Updated recommendations for the use of typhoid vaccine-Advisory Committee on Immunization Practices, United States, 2015. MMWR Morb Mortal Wkly Rep 2015;64:305-308. 